\title{
Configurações
}

Revista de sociologia

\section{Responsabilidad social y territorio: Hoteles de tres estrellas ubicados en la Eurorregión}

Social responsibility and territory: three stars hotels located in the Euroregion Responsabilité sociale et territoire: hôtels de trois étoiles situé dans l'Euroregion

\section{María Dolores Sánchez-Fernández}

\section{OpenEdition}

\section{Journals}

Edición electrónica

URL: http://journals.openedition.org/configuracoes/1832

DOI: $10.4000 /$ configuracoes. 1832

ISSN: 2182-7419

\section{Editor}

Centro de Investigação em Ciências Sociais

Edición impresa

Fecha de publicación: 1 junio 2013

Paginación: 143-160

ISBN: 1646-5075

ISSN: 1646-5075

\section{Referencia electrónica}

María Dolores Sánchez-Fernández, « Responsabilidad social y territorio: Hoteles de tres estrellas ubicados en la Eurorregión », Configurações [En línea], 11 | 2013, Puesto en línea el 22 septiembre 2014, consultado el 01 mayo 2019. URL : http://journals.openedition.org/configuracoes/1832 ; DOI : 10.4000/configuracoes. 1832

Este documento fue generado automáticamente el 1 mayo 2019. 


\section{Responsabilidad social y territorio: Hoteles de tres estrellas ubicados en la Eurorregión}

Social responsibility and territory: three stars hotels located in the Euroregion Responsabilité sociale et territoire: hôtels de trois étoiles situé dans l'Euroregion

María Dolores Sánchez-Fernández

\section{Introducción}

1 El sector turismo tiene varias características que lo convierten en una prioridad en el área geográfica de Galicia y Norte de Portugal dada su importancia como factor de desarrollo y motor de crecimiento económico. Estas dos regiones pertenecen a dos países fronterizos con una cultura similar aunque diferentes desde el punto de vista institucional y legislativo. El factor cultural influye en el grado de implantación de las prácticas socialmente responsables (Fanjul, 2010). Creemos que su estudio es interesante debido a las decisiones adoptadas por las instituciones de ambos países, así como la evolución de la normativa, hecho que puede afectar a la práctica de la Responsabilidad Social Corporativa (RSC) según el área geográfica. Uno de los ejes vertebradores en la política de turismo es en la actualidad la determinación de la oferta turística basada en la estrategia diferenciada de la calidad. Esta impregna un nuevo modelo de gestión en que las organizaciones consiguen sus objetivos de creación de valor bajo las tres dimensiones social, económica y medioambiental, relacionándose con las partes interesadas de forma diferente. Desde el año 2007 se producen recortes de todo tipo en ambas regiones, debido a la crisis económica, momento que es propio para vislumbrar el comportamiento socialmente responsable o irresponsable de las empresas. El papel de los hoteles es clave en el impacto sobre el entorno y sus grupos de interés, tanto en la oferta de sus servicios como la ubicación de sus instalaciones. Es importante que los profesionales integrados en estas entidades se responsabilicen de las consecuencias e impactos derivados del desarrollo de sus actividades. 
2 La estructura de este artículo es la siguiente, en primer lugar desarrollaremos las connotaciones del comportamiento socialmente responsable (CSR) de las organizaciones. Seguidamente expondremos de forma más detallada la escala utilizada para la medición del grado de desarrollo de prácticas de RSC y a continuación, desarrollaremos el estudio estadístico descriptivo de las diferentes variables que conforman las tres dimensiones de la RSC y el análisis factorial de cada uno de los constructos. Finalmente expondremos las conclusiones extraídas en la investigación y la bibliografía.

\section{El comportamiento socialmente responsable de la empresas}

3 Analizamos en este epígrafe los diversos factores que pueden influir en la adopción de un CSR por parte de las organizaciones. La adopción de un CSR implica el desarrollo más o menos intenso de prácticas de RSC en la organización. Las empresas adoptan este comportamiento en función del país en que se encuentren ubicadas, debido a la implicación mayor o menor de las instituciones, éstas pueden influenciar a las empresas de forma más o menos intensa (Galaskiewicz, 1991; Maigan y Ralston, 2002 y Campbell, 2007).

4 Martín, et al. (2008) identifican que las compañías adquieren un papel cada vez más relevante en la transformación tanto económico, ambiental como social en las áreas geográficas donde ejercen su ámbito de maniobra. Por lo que este hecho influye que de forma más intensa se tiene en consideración el impacto que las organizaciones generan en su entorno. En consecuencia el concepto de la RSC adquiere un papel más relevante, traspasando el marco de las herramientas de gestión de las propias empresas, alcanzando un nuevo rol y afectando a la percepción de los diferentes grupos de interés que se relacionan con la misma.

Además, las empresas que operan en diferentes países difunden sus prácticas, entre ellas las de RSC. Nieto (2008) identifica a los grupos de interés como una parte influyente en la adopción de prácticas de RSC. Uno de los motivos por los cuales este tipo de organizaciones adoptan criterios de RSC es por la presión que ejercen los grupos de interés con el fi $\mathrm{n}$ de contrarrestar efectos negativos que se puedan producir. La consecuencia inmediata de estos hechos es la colaboración de la difusión de las prácticas de RSC en los entornos en los que la empresa desarrolla su actividad. Las prácticas de RSC se llevan a cabo en las diferentes áreas geográficas con mayor o menor intensidad en función de la cultura, la promoción por parte de las instituciones, la afectación de normas y legislación que afecta a esa área geográfica y la tipología de empresas que desarrollan sus actividades en esa área geográfica.

6 Establecido el vínculo entre RSC y territorio estudiaremos la conceptualización de la RSC y bajo qué perspectivas se incorporan en las empresas. El concepto de RS está en continua evolución a lo largo del tiempo, adaptándose a las preocupaciones del momento (transparencia, ética, derechos humanos, entre otros); ajustándose a las características para lograr incentivar a las empresas (voluntaria, obligatoria) y en función de la especialización de la materia a tratar (universitaria, organizativa). Hoy en día concurre una gran proliferación de conceptos, teorías, enfoques y terminologías relacionadas con la temática de la responsabilidad social de las empresas (ciudadanía corporativa, rendición de cuentas corporativa, sostenibilidad corporativa, entre otros) (Garriga y Melé, 
2004). Dahlsurd (2008) presenta 37 definiciones de RSC propuestas por instituciones que promueven esta área, de ellas se extrae que la mayoría de las definiciones aportadas se refieren a las partes interesadas, a aspectos sociales y económicos, así como la voluntariedad, quedando los aspectos medioambientales como referencias menos frecuentes. El concepto de la RSC hoy en día sigue siendo un concepto controvertido (Claasen y Roloff, 2012). Cada uno de los grupos de interés tiene una visión diferente e interpreta la RSC de forma distinta: participación de la acción coordinada entre las partes interesadas (ONG); ventaja competitiva (asociaciones empresariales); recelo como posible sustituto de las formas de negociación colectiva (sindicatos). En la actualidad la responsabilidad social en las empresas se incorpora en la perspectiva de los negocios bajo el denominado Triple Resultado (Elkington, 1997; Papmehl, 2002; Norman y MacDonald, 2003; Brown, Dillard y Scott, 2006; Colbert y Kurucz, 2007) en el que se incluyen las tres dimensiones la económica, la medioambiental y la social. El campo de la responsabilidad social corporativa ha evolucionado en la disciplina de administración (Gerde y Wokutch, 1998; Rowley y Berman, 2000; De Bakker, Groenewegen y DenHond, 2005; Lockett, Moon y Visser, 2006; Bigné, Currás y Rivera, 2010), no perdiendo su carácter eminentemente interdisciplinar (Perdomo y Escobar, 2011).

7 Cabe destacar que, Campbell (2007); Nieto (2008) y Godos y Fernández (2011) identifica a los directivos de las organizaciones como grupos muy influyentes en la adopción de prácticas de responsabilidad social. A este grupo se le convence bien argumentando que realizan lo correcto, lo éticamente correcto o bien convenciéndolos que les sirve alcanzar sus propios intereses la implantación de la RSC en la organización Campbell (2007). Por ello diversos estudios toman como informantes de lo que sucede en la empresa a los directivos de las organizaciones con el fi n de recoger la información de las prácticas de RSC.

\section{Escala RSC}

8 Aupperle, Carrol y Hatfi el (1985); Singhapakdi et al (1996); Quazi y O’Brien (2000); Martín et al (2008); Gallardo, Sánchez y Corchuelo (2011); Perdomo y Escobar (2011) y GallardoVazquez y Sánchez-Hernández (2012) han desarrollado distintos instrumentos de medida de RSC bajo diferentes enfoques. Aupperle, Carrol y Hatfi el (1985) desarrollaron una escala para medir los valores individuales y las actitudes hacia la RSC, por parte de los directivos, en base al modelo de las cuatro dimensiones de Carroll (1979). Ruf, Muralidhar y Paul (1998) desarrollaron una escala para captar la naturaleza multidimensional de la RSC. Singhapakdi et al. (1996) diseñaron una escala que permite medir de la ética y la RSC, denominada PRESOR (Perceived Role of Ethics and Social Responsibility), orientada a la medición de los valores individuales de los directivos. En base a los estudios previos desarrollados por Davis (1973) y Orpen (1987), Quazi y O'Brien (2000) elaboraron una escala que permite medir de las actitudes ante la responsabilidad social.

9 Para la medición de la RSC en el sector turístico, Martín et al (2008) han elaborado una escala compuesta por diferentes apartados en base a las tres dimensiones de la responsabilidad social. Escala laboriosa y extensa, dirigida a los gerentes de los puertos deportivos. Gallardo, Sánchez y Corchuelo (2011) y Gallardo-Vázquez y SánchezHernández (2012) articulan una escala de RSC que validan en torno la perspectiva de la triple dimensión (Elkington, 1997). Esta escala esta enriquecida con aspectos que amplían la visión de la organización. El cuestionario está orientado a los directores/as de las 
empresas pertenecientes a diferentes sectores, entre los que se incluye el turismo. Es una escala con una extensión adecuada y asequible en la que se obtiene información en base a una escala likert de las prácticas de RSC desarrolladas por estas organizaciones. La escala se elabora encaminada al ámbito de hacia la gestión empresarial. Encontramos esta última escala ideal para incorporar en nuestro estudio orientado a la gestión, basado en la triple dimensión y dispone de un tamaño adecuado para incorporarla como escala de medición entre otros constructor, por ello la utilizaremos en nuestra investigación.

Hemos adaptado la escala para nuestro caso de estudio, utilizando una escala Likert de cinco puntos, donde 1 es "totalmente en desacuerdo" y 5 "totalmente de acuerdo". La escala ha sido validada previamente para el tejido empresarial contenido en el área geográfica de la Comunidad de Extremadura, España. La aplicación de esta escala en nuestro estudio abarca mayor área geográfica, más concreta en cuanto al sector y unidad objeto de estudio, los hoteles de tres estrellas ubicados en las regiones Galicia (España) y Norte de Portugal (Portugal).

\section{Análisis de las prácticas de RSC}

\subsection{Introducción}

11 En este apartado exponemos en primer lugar la unidad objeto de estudio seguido de la metodología utilizada para llevar a cabo el análisis de las prácticas de RSC. A continuación desarrollamos análisis estadístico descriptivo del comportamiento de los datos pertenecientes a los subconstructos de las tres dimensiones de la RSC. Finalizando este apartado con la exposición del análisis factorial de los constructos de las tres dimensiones social, económica y medioambiental.

12 La unidad objeto de estudio son los hoteles de tres estrellas ubicados en Galicia y en el Norte de Portugal. Nos hemos centrado en los hoteles de tres estrellas dado que creemos que este tipo de hoteles podrán relacionarse más con las prácticas de responsabilidad social que los hoteles de una y dos estrellas. Este categoría de hoteles implantan prácticas de RS con la suficiente dimensión, entidad y fundamentos para poder recoger información. El número de hoteles de tres estrellas contabilizado es suficiente para poder obtener una muestra adecuada permitiéndonos obtener una visión homogénea seleccionando organizaciones de la misma categoría. Los hoteles que más se resienten económicamente a lo largo de este periodo de crisis son los de tres estrellas frente a los de cuatro y cinco estrellas. Este hecho conlleva a que estén expuestos a que su CSR sea más inestable debido al alto descenso de recursos. Además los hoteles de esta categoría suelen ser más independientes, no incorporando las directrices generales de RSC provenientes de una cadena hotelera, ya que tienen más propensión a pertenecer a pequeños propietario. Los servicios añadidos para alcanzar la categoría de tres estrellas son inferiores para alcanzar la categoría de cuatro y cinco estrellas, lo que puede llevar a que sean más selectivos a implantar unas y otras prácticas de RSC.

La muestra recogida y analizada en este trabajo corresponde al 30\% de la población. La encuesta se dirigió a los directores/as de los hoteles, lo que supone una propia limitación en la investigación ya que recogemos información de tan solo un informante. En primer lugar se comenzó la fase pretest en el mes de abril de 2012 mediante formulario web. Posteriormente, se recogieron los datos en el periodo comprendido entre mayo y agosto de 2012 mediante el envío por correo electrónico del cuestionario en formato doc y 
formulario electrónico; visitas de forma presencial; fax; correo postal ordinario y contacto telefónico.

14 Las variables bajo estudio son los ítems contenidos en la escala de RSC de Gallardo, Sánchez y Corchuelo (2011) y Gallardo-Vázquez y Sánchez-Hernández (2012). Para su medición se utilizó una escala tipo Likert, de 1 a 5 , correspondiendo al nivel más bajo y el nivel más alto, señalando si estaban en desacuerdo o de acuerdo con las afirmaciones plasmadas en el cuestionario.

15 Los datos se someten a análisis factoriales, de correlaciones, a pruebas de fiabilidad y análisis estadístico descriptivo para cuya realización se emplea el paquete estadístico SPSS (versión 18.0). A continuación, en primer lugar mostraremos los resultados del análisis estadístico descriptivo. Se pretende validar la escala con el objeto de poder extraer el conjunto adecuado de ítems que conformen los constructos con el fi $\mathrm{n}$ de poder ser aplicados y comparados en futuros estudios con una sólida base.

\subsection{Análisis estadístico descriptivo}

En este apartado desarrollaremos el análisis estadístico descriptivo de los datos pertenecientes a los tres subconstructos que conforman la RSC, los cuales exponemos en las Tablas 1 a 2.

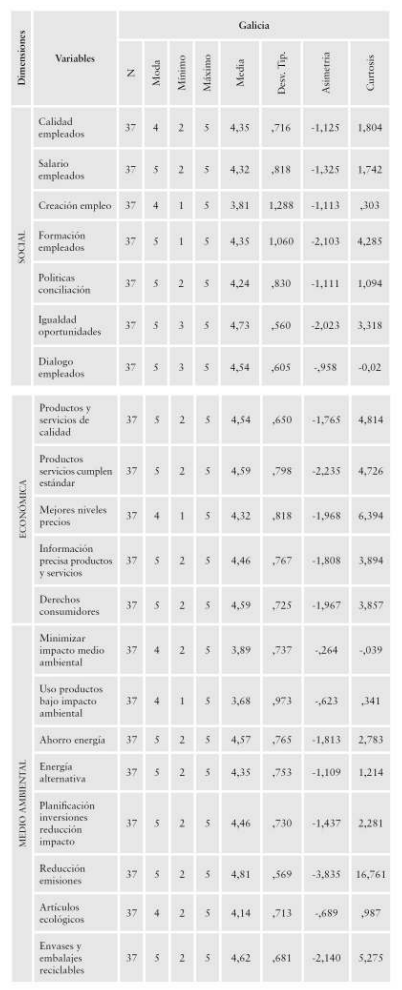

Tabla 1. Estadísticos descriptivos según Dimensiones de RSC de hoteles de tres estrellas de Galicia

FUENTE: ELABORACIÓN PROPIÁ 


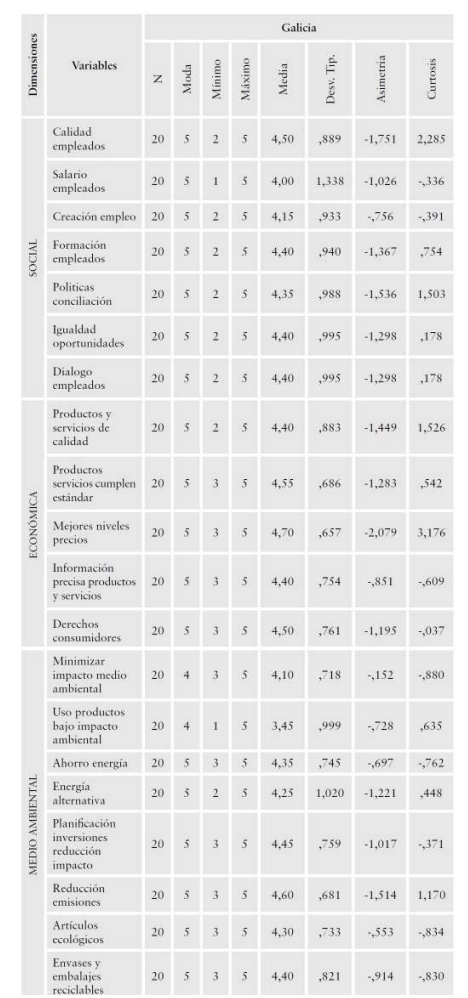

Tabla 2. Estadísticos descriptivos según Dimensiones de RSC de hoteles de tres estrellas del Norte de Portugal

Fuente: Elaboración propia

17 A continuación analizaremos los datos expuestos en las Tablas 1 y 2. Se puede observar que en la dimensión social, la existencia de igualdad de oportunidades para todos los empleados es la práctica más desarrollada por los hoteles pertenecientes a Galicia, siendo la preocupación por mejorar la calidad de vida de los empleados la práctica más utilizada por los hoteles de la Región Norte de Portugal. El compromiso con la creación de empleo (aceptación de becarios, creación de nuevos puestos, etc) es la práctica menos desarrollada por los hoteles en Galicia, siendo la que menos se practica en la Región Norte la relación del salario de los empleados con las competencias y los rendimientos que obtienen. El resto de las prácticas de responsabilidad social pertenecientes a la dimensión social se mantienen más o menos en el mismo nivel de desarrollo.

En el análisis de las prácticas más utilizadas encuadradas en la dimensión económica, podemos observar que ésta es la más equilibrada en su desarrollo, no produciéndose una diferencia excesiva en la mayor utilización entre las diferentes prácticas, pero manteniendo un nivel elevado de desarrollo de todas ellas. En Galicia, los hoteles desarrollan prácticas relacionadas con la preocupación porque los productos $\mathrm{y} / \mathrm{o}$ servicios cumplan los estándares nacionales e internacionales de calidad así como tienen como eje prioritario en su gestión el respeto a los derechos de los consumidores. La práctica menos desarrollada por los hoteles de Galicia es la distinción por mantener los mejores niveles de precios en relación con la calidad ofrecida, a diferencia de los hoteles ubicados en la Región Norte de Portugal, éstos la identifican como la práctica que desarrollan con mayor intensidad. La práctica menos utilizada por los hoteles portugueses es el proporcionar a los clientes información completa y precisa sobre sus productos y/o servicios. 
19 Analizando las prácticas pertenecientes a la dimensión medioambiental, se deduce que es la dimensión que mayor variabilidad de desarrollo presenta en ambas zonas geográficas. Tanto los hoteles de Galicia como los ubicados en la Región Norte de Portugal la utilización de combustibles, productos en curso y/o transformados de bajo impacto ambiental es la práctica menos desarrollada por los hoteles de ambas zonas geográficas, no siendo en la misma intensidad, pero sí adoptando una media inferior a 4, además es la única práctica que han valorado utilizando el total de los rangos expuestos, de 1 a 5 en la dimensión medioambiental. Siendo la segunda práctica menos desarrollada la capacidad de minimizar el impacto medioambiental. La utilización de las prácticas menos desarrolladas están confrontadas con la práctica que identifican que más desarrollan en ambas regiones, aunque no en la misma intensidad, la posición que adoptan de estar a favor de la reducción de emisiones de gases, residuos y reciclaje de materiales.

En el análisis de la moda, el valor más frecuente en la matriz de datos en Portugal en todas las variables es 5 a excepción de las prácticas de la dimensión medioambiental relacionadas con la minimización del impacto ambiental y la utilización de productos de bajo impacto ambiental que es 4 . A diferencia de los hoteles ubicados en Galicia, aunque presentan muchas de las prácticas una valoración de 5 , existen prácticas en las diferentes dimensiones con una valoración igual a 4. En ambas regiones no se encuentra ninguna variable con el valor más frecuente en las matrices de datos inferior a 4, por lo tanto, manifiestán los directores de hotel que de forma intensa desarrollan prácticas de RSC. Los hoteles ubicados en Portugal desarrollan prácticas de forma intensa en todas las dimensiones, disponiendo de un par de prácticas en la dimensión medioambiental que no practican tan intensamente, a diferencia de los hoteles ubicados en Galicia, en la que nos encontramos que desarrollan prácticas de manera más o menos intensa, en cada una de las dimensiones de la RSC.

21 Las prácticas desarrolladas en las dimensiones social, económica y medioambiental en Galicia y en la Región Norte de Portugal se comportan de forma asimétricamente negativa, los valores se tienden a reunirse de forma más intensa en la parte derecha de la media. La variable que mayor distancia la separa de aglomeración de los valores con respecto a la media son las variables, la práctica asimilada a la existencia de igualdad de oportunidades para todos los empleados, concretamente es la más valorada desarrollada por los hoteles de Galicia en la dimensión social. Igualmente en Portugal la práctica más desarrollada es la más distanciada, la preocupación por mejorar la calidad de vida de los empleados. El mismo comportamiento se percibe en las otras dos dimensiones, las prácticas más desarrolladas por los hoteles se encuentran más distanciadas. Con referencia a la normalidad, teniendo en cuenta los coeficientes de asimetría y el coeficiente de curtosis, ninguno de los datos pertenecientes al rango correspondiente a los hoteles de Galicia y del Norte de Portugal de las diferentes variables se distribuyen normalmente.

Del análisis estadístico descriptivo podemos extraer que se manifiesta una tendencia del comportamiento ante las diferentes dimensiones de la responsabilidad social corporativa. El desarrollo de prácticas de responsabilidad social en las dimensiones social y económica es diferente, pero es similar en las prácticas utilizadas en la dimensión medioambiental. La dimensión más valorada es la dimensión económica mostrando en segundo lugar la social y el tercer lugar lo ocupa la medioambiental por los hoteles ubicados en Galicia. Los hoteles pertenecientes a la Región Norte de Portugal desarrollan más las prácticas de la 
dimensión económica y en segundo lugar las prácticas relacionadas con la dimensión social y medioambiental.

\subsection{Análisis factorial}

En primer lugar detallaremos las premisas en las que hemos basado nuestro estudio y seguidamente, trataremos el análisis factorial en función de las diferentes dimensiones de la RSC. Los datos se someterán a los siguientes criterios expresados en la Tabla 3.

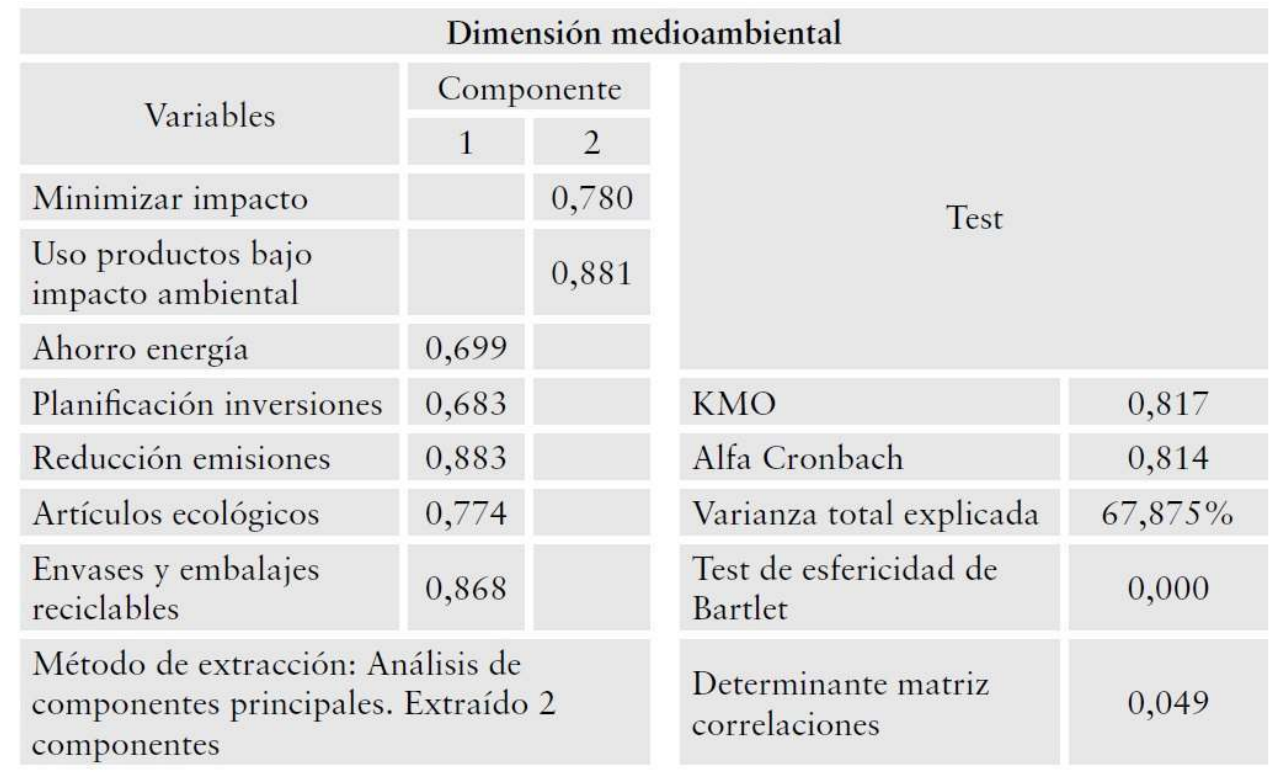

Tabla 3. Criterios pruebas de unidimensionalidad de los factores

Fuente: Elaboración propia

Procedemos al análisis factorial de cada uno de los tres constructos que conforman las prácticas de RSC.

\subsubsection{Análisis del Constructo de la Dimensión Medioambiental}

Las variables que conforman la dimensión medioambiental de la RSC, en su inicio, son las siguientes: Minimizar impacto ambiental (Minimpmed); Usar productos de Bajo impacto ambiental (Pbajoimpme); Ahorro de Energía (Ahorrenerg); Fuentes de Energía Alternativas (Energaltern); Planificación de inversiones de reducción impacto (Planinvrimp); Reducción de emisiones (Reducemis); Artículos Ecológicos (Articecolog) y Envases y embalajes reciclables (Envembrecicl).

El constructo dimensión medioambiental cumple todos los requisitos (Tabla 3), tras someter los datos a una segunda fase de las pruebas de análisis factorial. Finalmente en nuestro caso la escala de la dimensión medioambiental se compone de los dos factores en los que se incluyen dos y cinco ítems, tras la eliminación el ítem denominado fuentes de energía alternativa. Finalmente, en la Tabla 4 incluimos las variables que componen este constructo, así como los principales resultados de las pruebas a las cuales hemos sometido los datos. 


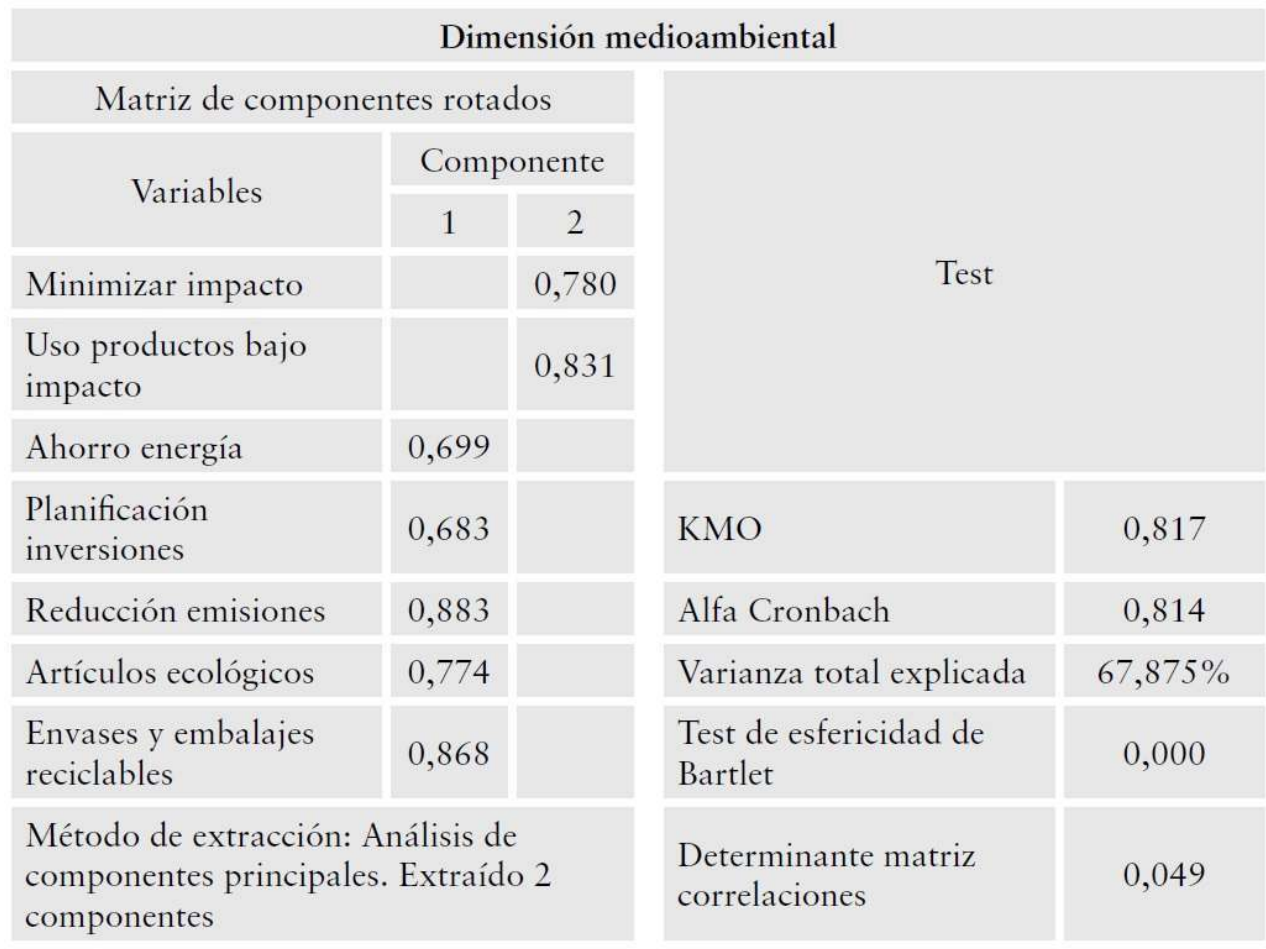

Tabla 4. Información del constructo Dimensión Medioambiental

Fuente: Elaboración propia

\subsubsection{Análisis del constructo Dimensión Económica}

Las variables que conforman la dimensión económica de la RSC, en su inicio, son las siguientes: Productos y servicios de calidad (prodservq); Productos y servicios cumplen un estándar (prodservcstd); Mejores niveles de precios (mejnivprec); Información precisa de productos y servicios (prodservinfo) y Derechos de los consumidores (derchconsu).

El constructo de la dimensión económica de prácticas de RSC está constituido por un solo factor, en el cual se incluyen la totalidad de las variables propuestas inicialmente, dando cumplimiento a todos los criterios previamente establecidos (Tabla 3). Finalmente, en la Tabla 5 incluimos las variables que componen esta escala y sus principales resultados. 


\begin{tabular}{|c|c|c|c|}
\hline \multicolumn{4}{|c|}{ Dimensión económica } \\
\hline \multicolumn{2}{|c|}{ Matriz de componentes } & \multirow{4}{*}{ Test } & \\
\hline \multirow{2}{*}{ Variables } & Componente & & \\
\hline & 1 & & \\
\hline $\begin{array}{l}\text { Productos y servicios } \\
\text { de calidad }\end{array}$ & 0,866 & & \\
\hline $\begin{array}{l}\text { Productos y servicios } \\
\text { estándar }\end{array}$ & 0,748 & $\mathrm{KMO}$ & 0,795 \\
\hline Mejores niveles precios & 0,839 & Alfa Cronbach & 0,863 \\
\hline $\begin{array}{l}\text { Información productos } \\
\text { y servicios }\end{array}$ & 0,785 & Varianza total explicada & $65,139 \%$ \\
\hline Derechos consumidores & 0,792 & $\begin{array}{l}\text { Test de esfericidad de } \\
\text { Bartlet }\end{array}$ & 0,000 \\
\hline \multicolumn{2}{|c|}{$\begin{array}{l}\text { Método de extracción: Análisis de } \\
\text { componentes principales. Extraído } 1 \\
\text { componente }\end{array}$} & $\begin{array}{l}\text { Determinante matriz } \\
\text { correlaciones }\end{array}$ & 0,087 \\
\hline
\end{tabular}

Tabla 5. Información del constructo Dimensión Económica

Fuente: Elaboración propia

\subsubsection{Análisis del Constructo de la Dimensión Social}

Las variables que conforman la dimensión social de la RSC, inicialmente son las siguientes: Calidad de empleados (calidemp); Salario empleados (salaremp); Creación de empleo (creacemp); Formación empleados (formemp); Políticas de conciliación (politconc); Igualdad de oportunidades (igualopor); Mecanismos de diálogo de los empleados (dialgemp).

En el constructo dimensión social está conformado por un solo factor en el que se incluyen seis ítems. Esta escala cumple todos los criterios (Tabla 3) tras someter los datos a las pruebas en una segunda fase en el que tan solo se elimina el ítem creación de empleo. Dada la situación actual es lógico que este ítem se elimine, la tasa de paro desde el año 2007 ha sufrido un incremento vertiginoso alcanzando en España un 25\% y en Portugal un 17\%. Finalmente, en la Tabla 6 incluimos los principales resultados del factor y las variables que componen el constructo prácticas RSC en la dimensión social: 


\begin{tabular}{|c|c|c|c|}
\hline \multicolumn{4}{|c|}{ Dimensión social } \\
\hline \multicolumn{2}{|c|}{ Matriz de componentes } & \multirow{5}{*}{ Test } & \\
\hline \multirow{2}{*}{ Variables } & Componente & & \\
\hline & 1 & & \\
\hline Calidad empleados & 0,796 & & \\
\hline Salario empleados & 0,799 & & \\
\hline Formación empleados & 0,710 & $\mathrm{KMO}$ & 0,778 \\
\hline Politicas conciliación & 0,734 & Alfa Cronbach & 0,866 \\
\hline Igualdad oportunidades & 0,822 & Varianza total explicada & $61,527 \%$ \\
\hline Dialogo empleados & 0,838 & $\begin{array}{l}\text { Test de esfericidad de } \\
\text { Bartlet }\end{array}$ & 0,000 \\
\hline $\begin{array}{l}\text { Método de extracción: A } \\
\text { componentes principales. } \\
\text { componente }\end{array}$ & $\begin{array}{l}\text { álisis de } \\
\text { Extraído } 1\end{array}$ & $\begin{array}{l}\text { Determinante matriz } \\
\text { correlaciones }\end{array}$ & 0,050 \\
\hline
\end{tabular}

Tabla 6. Información del constructo Dimensión Social

Fuente: Elaboración propia

\section{Conclusiones}

De los argumentos teóricos podemos extraer que el papel de las instituciones es fundamental para que las empresas adopten una actuación activa orientada al comportamiento socialmente responsable. Las organizaciones implementan este comportamiento en función de la implicación más o menos intensa de las instituciones (Maigan y Ratlson, 2002) y en función de si crean o no un conjunto de adecuados incentivos (Galaskiewicz, 1991). Considera Campbell (2007) que las instituciones son un elemento clave para limitar o motivar a las organizaciones. Además las instituciones pueden llegar a influir en el ápice estratégico (Maigan y Ratson, 2002). Los factores que influyen en que las organizaciones adopten un comportamiento socialmente responsable son la ubicación de las instalaciones, los países donde operan y la relación de incentivos por parte de las instituciones.

Otra de las consideraciones a tener en cuenta en la influencia del comportamiento de las empresas es el proceso de internacionalización, en el cual muchas de ellas se ven inmersas. Este progreso ayuda a la difusión de prácticas de responsabilidad social en las organizaciones a lo largo de los diferentes países en los que operan (Nieto, 2008).

En el análisis estadístico descriptivo podemos extraer que se manifiesta una tendencia diferente ante el comportamiento asociado a las diferentes dimensiones de la responsabilidad social corporativa. El desarrollo de prácticas de responsabilidad social en las dimensiones social y económica es diferente, pero es similar en las prácticas utilizadas en la dimensión medioambiental. En este caso tanto en España como en Portugal tienen desarrollada con gran amplitud legislación orientada al medioambiente bajo el paraguas de la Unión Europea. En el caso de materia social la legislación y normativa es diferente, en el caso de de Galicia se promocionan cuestiones de carácter social relacionadas con igualdad de oportunidades con menor intensidad a las realizan en Portugal. En Galicia se deja de lado cuestiones relacionadas con el empleo, ya que la situación de crisis 
económica hace que se creen menos empleos y las condiciones se devalúen, cuestión que también ocurre en Portugal pero existe mayor colaboración y sensibilidad en este sentido. En el ámbito económico tanto en Galicia como en Portugal preocupa todo aquello relacionado con los intereses de la dimensión económica.

Se ha realizado el análisis de las prácticas de responsabilidad social corporativa bajo la hipótesis de la afirmación extraída de Maigan y Ralston (2002), la tendencia al comportamiento socialmente responsable varía en función de diferentes países. Se confirma la propensión de un comportamiento diferente según los países en las dimensiones social y económica en los hoteles de tres estrellas pertenecientes a la región Norte de Portugal y Galicia. Estos hoteles presentan una tendencia a un comportamiento socialmente responsable similar en la dimensión medioambiental. En el anterior apartado hemos expuesto las principales motivaciones.

35 A partir del análisis factorial confirmatorio con rotación Varimax, las variables que forman parte de la escala de RSC, en base a las tres dimensiones para los hoteles de tres estrellas ubicados en Galicia y Norte de Portugal es muy similar a las extraídas para el tejido empresarial que conforma la Comunidad de Extremadura. Por lo que se propone utilizar en futuros estudios esta escala de RSC para comprobar si el comportamiento es similar en otros países, así como confirmar si se mantienen el mismo conjunto de ítems para cada uno de los factores.

En nuestro estudio nos hemos apoyado en medidas basadas en las percepciones de los Directores/as de los hoteles, por lo que en todos los casos la información del hotel se recibió de un único informante. Hemos de admitir la posibilidad de que las percepciones de los encuestados no se correspondan del todo con la realidad, y que para el desarrollo de un estudio más completo debería tenerse en cuenta la opinión de otros grupos de interés tanto internos como externos a la organización.

En las futuras líneas de investigación se propone realizar una comparativa entre los hoteles de tres estrellas y los de mayor categoría, ya que en este estudio supone una propia limitación la unidad de análisis. Para que se puedan extraer las conclusiones generales orientadas a todo el territorio nacional, sería de gran interés comparar si la tendencia que se enmarca en este estudio se cumple para todo el territorio a nivel España y Portugal, extraer las diferencias y similitudes entre ambos países y la comparativa de los resultados aportados en la presente investigación.

Por otra parte, dada la situación coyuntural de la economía en el momento que se realiza esta investigación, se propone realizar este mismo estudio en un momento económico más favorable, pudiendo comparar en dos períodos económicos diferentes si afecta a un comportamiento más o menos responsable e identificar si se cumple para una o todas las dimensiones de la RSC.

\section{Agradecimientos}

Agradecer los comentarios plasmados por los árbitros asignados a la lectura de mi texto para la consideración en la Revista Configurações. 


\section{BIBLIOGRAFÍA}

AUPPERLE, K. E.; CARROLL, A. B. y HATFIELD, J. D. (1985), “An Empirical Examination of the Relationship between Corporate Social Responsibility and Profitability", Academy of Management Journal, 28(2): 446-463.

BARTLETT, M. S. (1950), “Tests of significance in factor analysis”, British Journal of Psychology, 3: 77-85.

BARTLETT, M. S. (1951), "A further note on tests of signifi cance in factor analysis”, British Journal of Psychology, 4: 1-2.

BIGNÉ, E.; ALVARADO, A.; CURRÁs, R. y RIVERA, J. (2010), “Latest evolution of academic research in corporate social responsibility: An empirical análisis", Social Responsibility Journal, 6: 332-344.

BROWN, D., DILLARD, J. y SCOTT MARSHALL, R. (2006), “Triple Bottom Line: A Business Metaphor for a Social Construct", Documento de Trabajo, Universidad Autónoma de Barcelona.

CAMPBELL, J. L. (2007), “Why would corporations behave in socially responsible ways? An institutional theory of corporate social responsibility", Academy of Management Review, 32 (3):

946-967.

CARROLL, A.B. (1979), “A Three Dimensional Conceptual Model of Corporate Social Performance”, Academy of Management Review, 4(4): 497-505.

CLAASEN, C. y ROLOFF, J. (2012), "The Link Between Responsibility and Legitimacy: The Case of De Beers in Namibia”, Journal of Business Ethics, 107(3): 379-398.

COLBERT, B. A. y KURUCZ, E. (2007), “Three conceptions of Triple Bottom Line Business Sustainability and the Role for HRM”, HR, Human Resource Planning, 30(1): 21-29.

DAHLSRUD, A. (2008), "How corporate social responsibility is defi ned: An analysis of 37 definitions”, Corporate Social Responsibility and Environmental Management, 15: 1-13.

DAVIS, K. (1973), “The cases for and against business assumption of Social Responsibilities", Academy of Management Journal, 16(2): 312-322.

DÍEZ MARTÍN, F.; BLANCO GONZÁLEZ, A. y PRADO ROMÁN, C. (2010), “Medición de la legitimidad organizativa: El caso de las Sociedades de Garantía Recíproca”, Cuadernos de Economía y Dirección de la Empresa, 13(43): 115-143.

ELKINGTON, J. (1998), Cannibals with Forks: The Triple Bottom Line of 21st Century Business, (2 ed.), Oxford: Capstone Publishing Ltd.

FANJUL, E. (2010), “Factores culturales e internacionalización de la empresa”, ICE, 856: 7-19.

GALASKIEWICZ, J. (1991), “Making corporate actors accountable: Institution-building in Minneapolis-St. Paul”, in Walter W. Powell e Paul J. DiMaggio (eds.) The new institutionalism in organizational analysis. Chicago: University of Chicago Press: 293-310.

GALLARDO VÁZQUEZ, D.; SÁNCHEZ HERNÁNDEZ, M. I. e CORCHUELO MARTÍNEZ-AZÚA, M. B. (2011), "Validación de un instrumento de medida para la relación entre la orientación a la responsabilidad social corporativa y otras variables estratégicas de la empresa", Paper presentado en el Congreso XVI Congreso AECA, Septiembre, Granada. 
GALLARDO-VAZQUEZ, D. y SANCHEZ-HERNANDEZ, I. (2012), “Information on corporate social responsibility and SME's environmental responsiveness: A regional study", Economics and Sociology, 5 (2): 103-115.

DE BAKKER, F. G.; GROENEWEGEN, P. y DEN HOND, F. (2005), “A bibliometric analysis of 30 years of research and theory on Corporate Social Responsibility and Corporate Social Performance", Business \& Society, 44 (3): 283-317.

GARRIGA, E. y MELÉ, D. (2004), “Corporate Social Responsibility Theories: Mapping the Territory”, Journal of Business Ethics, 53(1/2): 51-71.

GERDE, V. W. y WOKUTCH, R. E. (1998), "25 years and going strong: A content analysis of the first 25 years of the social issues in management division proceedings", Business and Society, 37(4): 414-446.

LOCKETT, A.; MOON, J. y VISSER, W. (2006), “Corporate social responsibility in management research: Focus, nature, salience and sources of influence", Journal of Management Studies, 43 (1): 115-136.

MAIGAN, I. e RALSTON, D. A. (2002), “Corporate social responsibility in Europe and the U.S.: Insights from businesses' self-presentations”, Journal of International Business Studies, 33 (3): 497-514.

MARTÍN ROJO, I.; GASPAR GONZÁLEZ, A. I.; CARO GONZÁLEZ, F. J.; CASTELLANOS VERDUGO, M. y OVIEDO GARCIA, M. A. (2008), La responsabilidad social corporativa en los puertos deportivos y clubes náuticos de Andalucía: Diagnóstico y propuestas de mejoras para la innovación turística, Sevilla: C. y D, Consejería de Turismo.

NIETO ANTOLÍN, M. (2008), “La difusión de las prácticas de responsabilidad social en las empresas multinacionales", Pecvinia, 1 (extra): 33-64.

NORMAN, W. y MCDONALD, C. H. (2003), “Getting to the Bottom of "Triple Bottom Line”. Business Ethics Quarterly, 14(2): 243-262.

NUNNALLY, J. C. (1978). Psychometric theory (2nd ed.). Nova Iorque: McGraw-Hill.

ORPEN, C. (1987), "The attitudes of United States and South African Managers t Corporate Social Responsibility", Journal of Business Ethics, 6(2): 89-96.

PAPMEHL, A. (2002), “Beyond the Gaap”, CMA Management, 76(5): 20-25.

PERDOMO, J. y ESCOBAR, A. (2011), “La investigación en RSE: una revisión desde el management”, Cuadernos de Administración, 24(43): 193-219.

PÉREZ LÓPEZ, C. (2005), Métodos estadísticos avanzados con Spss, Madrid, Thomson.

QUAZI, A. M. y O'BRIEN, D. (2000), “An empirical test of a cross-national model of Corporate Social Responsibility”, Journal of Business Ethics, 25: 33-51.

ROWLEY, T. (1997), "Moving beyond dyadic ties: a network theory of stakeholder influences", Academy of Management Review, 22: 887-910.

SINGHAPAKDI, A.; VITELL, S. J.; RALLAPALLI, K. C. y KRAFT, K. L. (1996), “The perceived role of Ethics and Social Responsibility: a scale development", Journal of Business Ethics, 15: 1131- 1140.

VERDÚ, A. (2002), Relación entre flexibilidad y desempeño organizativo: una aproximación desde la perspectiva de la gestión de la calidad total. Tesis Doctoral: Universidad Granada.

WANG, C. H. (2005), "Constructing multivariate process capability indices for short-run production”, Int.J. Adv. Manuf. Technol., 26: 1306-1311. 
WUBNEH, M. A. (1987), "Multivariate analysis of socio-economic charasteristics of urba areas in Ethiopia", Afr. Urban Quaterly, 2: 425-433.

\section{RESÚMENES}

Uno de los objetivos de este trabajo es comprobar la fiabilidad de la escala de medida del factor principal que conforman las dimensiones de la Responsabilidad Social Corporativa (RSC). Se realiza el análisis factorial de las tres dimensiones clásicas teóricamente aceptadas en base la triple dimensión. La muestra la conforman hoteles de tres estrellas pertenecientes a las Regiones Norte de Portugal y Galicia. Se obtiene que, cada una de las dimensiones del factor de primer orden RSC incorporan indicadores fiables (Alfa de Cronbach $>0,8$ ). Otro de los objetivos enmarcados en este estudio es el análisis estadístico descriptivo del comportamiento socialmente responsable por parte de las unidades objeto de estudio.

One objective of this work is to test the reliability of the measurement scale main factor that make the dimensions of Corporate Social Responsibility (CSR). Factor analysis is performed on the three classical dimensions based theoretically accepted the triple dimension. The sample is composed on three star hotels belonging to the North of Portugal and Galicia. You get that, on each of the dimensions of the major factor RSC incorporate reliable indicators (Cronbach's alpha> 0.8). Another objective enshrined in this study is the descriptive analysis of socially responsible behavior on the part of the units under study

Un des objectifs de ce travail est de tester la fiabilité de l'échelle de mesure du facteur principal qui fait partie des dimensions de la Responsabilité Sociale des Entreprises (RSE). L'analyse factorielle est effectuée sur les trois dimensions classiques théoriquement acceptées conformemente aux triples dimensions. L'échantillon est composé par des hôtels de trois étoiles qui appartiennentaux régions Nord du Portugal et de Galice. Le résultat es que chacune des dimensions du facteur de premier RSE incorpore des indicateurs fiables (Alpha de Cronbach>0,8). Un autre objectif incluí dans cette étude est l>analyse statistique descriptive du comportement socialement responsable de la part des unités étudiées.

ÍNDICE

Mots-clés: responsabilité sociale des entreprises, trois dimensions, hôtels, Eurorégion

Palabras claves: RSC, triple dimensión, hoteles, Eurorregión

Keywords: CSR, triple bottom line, hotels, Euroregion

\section{AUTOR}

\section{MARÍA DOLORES SÁNCHEZ-FERNÁNDEZ}

Universidade da Coruña

msanchezf@udc.es 